\title{
Global stability analysis of hepatitis B virus dynamics [version
}

\section{1; peer review: 1 approved]}

\author{
Olajumoke Oludoun (iD1, Olukayode Adebimpe1, James Ndako (D2, \\ Oluwakemi E. Abiodun (D1, Babatunde Gbadamosi³, Benedicta B. Aladeitan (D1 \\ ${ }^{1}$ Department of Physical Sciences, LANDMARK UNIVERSITY, Omu-Aran, Kwara State, Nigeria \\ ${ }^{2}$ Department of Microbiology, LANDMARK UNIVERSITY, Omu-Aran, Kwara State, Nigeria \\ ${ }^{3}$ Department of Computer Sciences, LANDMARK UNIVERSITY, Omu-Aran, Kwara State, Nigeria
}

\author{
V1 First published: 28 May 2021, 10:429 \\ https://doi.org/10.12688/f1000research.52785.1 \\ Latest published: 21 Jan 2022, 10:429 \\ https://doi.org/10.12688/f1000research.52785.2
}

\section{Abstract}

This paper considers the impact of an acute individual's spontaneous clearance, recovery of a chronic individual with full immunity, and risk factor reduction on a hepatitis $B$ virus (HBV) model. The existence and the positivity solution of the model are established. The model threshold quantity is defined and sensitivity analysis is analyzed to demonstrate the effect of various parameters on the spread of the virus. The global stability analysis of the equilibrium is shown using Lyapunov and comparison theorem methods. Finally, computational simulation is presented to validate the analytical solution. The results show that treatment, spontaneous clearance and reduction of the risk factor are highly successful in transmitting and regulating HBV transmission. The effective measure of these parameters as substantiated by our simulations, providing an excellent control method of the transmissible infection of HBV.

\section{Keywords}

Hepatitis B, mathematical model, positivity and existence, global stability, sensitivity, Lyapunov method, simulation

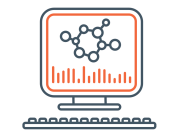

This article is included in the Bioinformatics

gateway.

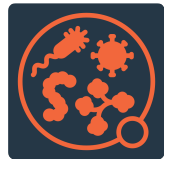

This article is included in the Pathogens

gateway.

\section{Open Peer Review}

\begin{tabular}{|c|c|c|c|}
\hline \multicolumn{4}{|c|}{ Approval Status $\checkmark \checkmark$} \\
\hline & 1 & 2 & 3 \\
\hline \multicolumn{4}{|l|}{ version 2} \\
\hline (revision) & & $\checkmark$ & $?$ \\
\hline 21 Jan 2022 & & view & view \\
\hline version 1 & $\checkmark$ & & \\
\hline 28 May 2021 & view & & \\
\hline
\end{tabular}

1. Abimbola Abolarinwa, University of Lagos, Lagos, Nigeria

2. Ran Zhang ID, University of Lagos, Lagos, Nigeria

3. Syafruddin Side ID, University of Lagos, Lagos, Nigeria

Any reports and responses or comments on the article can be found at the end of the article. 
Corresponding author: Olajumoke Oludoun (oludoun.olajumoke@Imu.edu.ng)

Author roles: Oludoun O: Conceptualization, Formal Analysis, Writing - Original Draft Preparation; Adebimpe O: Supervision, Writing Review \& Editing; Ndako J: Supervision; Abiodun OE: Methodology; Gbadamosi B: Software; Aladeitan BB: Writing - Original Draft Preparation

Competing interests: No competing interests were disclosed.

Grant information: The author(s) declared that no grants were involved in supporting this work.

Copyright: @ 2021 Oludoun O et al. This is an open access article distributed under the terms of the Creative Commons Attribution License, which permits unrestricted use, distribution, and reproduction in any medium, provided the original work is properly cited.

How to cite this article: Oludoun $\mathrm{O}$, Adebimpe $\mathrm{O}$, Ndako J et al. Global stability analysis of hepatitis B virus dynamics [version 1; peer review: 1 approved] F1000Research 2021, 10:429 https://doi.org/10.12688/f1000research.52785.1

First published: 28 May 2021, 10:429 https://doi.org/10.12688/f1000research.52785.1 


\section{Introduction}

Hepatitis B is a common liver infection caused by the potentially life-threatening hepatitis B virus (HBV). HBV can cause a serious infection, which places individuals at high risk of dying from fibrosis and cirrhosis of liver. It is a huge worldwide health issue. As reporting by the World Health Organization, around 360 million of the 2 billion people infected with the HBV are reported to have a lifelong chronic infection, and 887,000 of those individuals die from liver cirrhosis or primary hepatocellular carcinoma (WHO, 2020). As of 2016, 27 million individuals (10.5\%) of all people considered to be living with HBV were aware of their infection, while 4.5 million (16.7\%) of those diagnosed were receiving treatment (WHO, 2019). The Western Pacific region recorded the highest incidence rate of $\mathrm{HBV}$ at $6.2 \%$ of the adult population, while this was $6.1 \%$ in the African region, and $0.7 \%$ in the American region on (WHO, 2019). Although HBV lives outside the body for about seven days, it is still very possible for it to cause an infection if it is injected into an unvaccinated individual. It takes about 75 days on the average for the HBV to incubate but this can vary between 30 and 180 days. Detection of the virus can be between 30 and 60 days of being infected or consequently mature into full-blown HBV (CDC, 2019).

The HBV, a hepatotropic non-cytopathic virus, is responsible for the disease (Ribiero, 2002). In highly endemic areas, perinatal transmission or horizontal transmission (exposure to infected blood) are the primary means of transmission (Pan and Zhang, 2005). The most common method of transmission is from mother to child at birth, particularly from infected children to uninfected children within the first 5 years of life. Contaminated body fluids such as vaginal discharge, saliva, menstrual flow, and semen are other means of transmission (Pan and Zhang, 2005). Rarer means of transmission include transpiration, breast milk, sweat, and urine by percutaneous or mucosal exposure of infected individuals (Mpeshe and Nyerere, 2019). In particular, unvaccinated men who have sex with men and heterosexual people with several sexual partners or who have contact with sex workers may experience sexual transmission of HBV (Khan et. al, 2019). In less than $5 \%$ of cases, infections lead to chronic hepatitis in adulthood. Virus transmission can also occur either in health care facilities or through the reuse of needles and syringes among individuals who inject drugs. Furthermore, infection can occur during medical, surgical and dental procedures, by tattooing, or by using razors and similar products contaminated with infected blood (Mpeshe and Nyerere, 2019).

Typically, there is a 5-10\% chance of recovery for adults that develop chronic infections (Chenar et al., 2018). Host variables are believed to be responsible for determining whether the infection is cleared or becomes chronic, especially immune responses (Ciupe et al., 2007). Different aspects of HBV dynamics and the immune response during infection have been investigated by several mathematical models (Ribiero, 2002; Long et al., 2008; Lau et al., 2009; Wang et al., 2010; Qesmi et al., 2010; Pang et al., 2010).

As a result of the research mentioned above, we present an infectious disease model to better understand how testing and treatment affects HBV transmission dynamics and prevalence. The model formulation of HBV transmission dynamics, as well as the dynamical behavior of the model, including equilibria and stabilities is presented in this paper. The aim of this study is to contribute to the development of HBV control strategies and the establishment of intermediate objectives for intervention programs.

\section{Model formulation}

It has been clinically shown that a proportion of HBV acutely infected individuals can spontaneously clear the virus (Pan and Zhang, 2005). Also, infectious individuals under treatment can become prone to re-infection if they discontinue treatment, or consume alcohol or use of drugs, which can reduce the impact of the treatment. In view of this, the following model is developed where the population is divided into different states, namely: the susceptible, the acute, the chronic carriers, the treated and the recovered states.

At time $t$, denoted by $N(t)$, the total population is divided into the following five classes/subgroups (Table 1) corresponding to different epidemiological status.

$$
N(t)=S(t)+A(t)+C(t)+T(t)+R(t)
$$

where $S(t)$ are the susceptible populace, $A(t)$ is the populace that are acutely infected with HBV, $C(t)$ are the chronically/ clinically infected individuals, while $T(t)$ are individuals under treatment and $R(t)$ are the recovered classes.

Figure 1 schematically represents the epidemiology of HBV. The various disease stages are replicated by the various compartments (circle) and the arrows demonstrate the way an individual progress from one state to the other. It is assumed that at time $t$, susceptible individual $S$, enter the population at a constant rate $\zeta(1-\alpha)(1-\gamma C)$ where $\zeta$ is the birth rate, $\alpha$ is the proportion of population successfully immunized, while $\gamma$ is the probability that children born to carrier mothers will 
Table 1. Parameter descriptions.

\begin{tabular}{|l|l|}
\hline Parameter & Description \\
\hline$\zeta$ & birth rate \\
\hline$\alpha$ & Proportion of population successfully immunized \\
\hline$\lambda_{s}$ & Probability that children born to carrier mothers will develop to chronic state \\
\hline$\xi$ & Horizontal transmission coefficient \\
\hline$\eta$ & Reduced transmission coefficient \\
\hline $1 / \omega$ & Spontaneous clearance proportion \\
\hline$k$ & Duration of acute phase \\
\hline$\mu$ & Rate at which treated individuals recover with full immunity \\
\hline$\sigma$ & Natural death rate \\
\hline$v$ & Treatment rate for chronic individuals \\
\hline$\rho$ & Proportion of population recovering \\
\hline$\varepsilon$ & Duration of HBV treatment \\
\hline $\mathrm{S}(\mathrm{t})$ & Rate at which recovered population fall out from risk reduction \\
\hline $\mathrm{A}(\mathrm{t})$ & Susceptible Compartment \\
\hline $\mathrm{C}(\mathrm{t})$ & Acute Compartment \\
\hline $\mathrm{T}(\mathrm{t})$ & Chronic Compartment \\
\hline $\mathrm{R}(\mathrm{t})$ & Treatment Compartment \\
\hline & Recovered Compartment \\
\hline
\end{tabular}

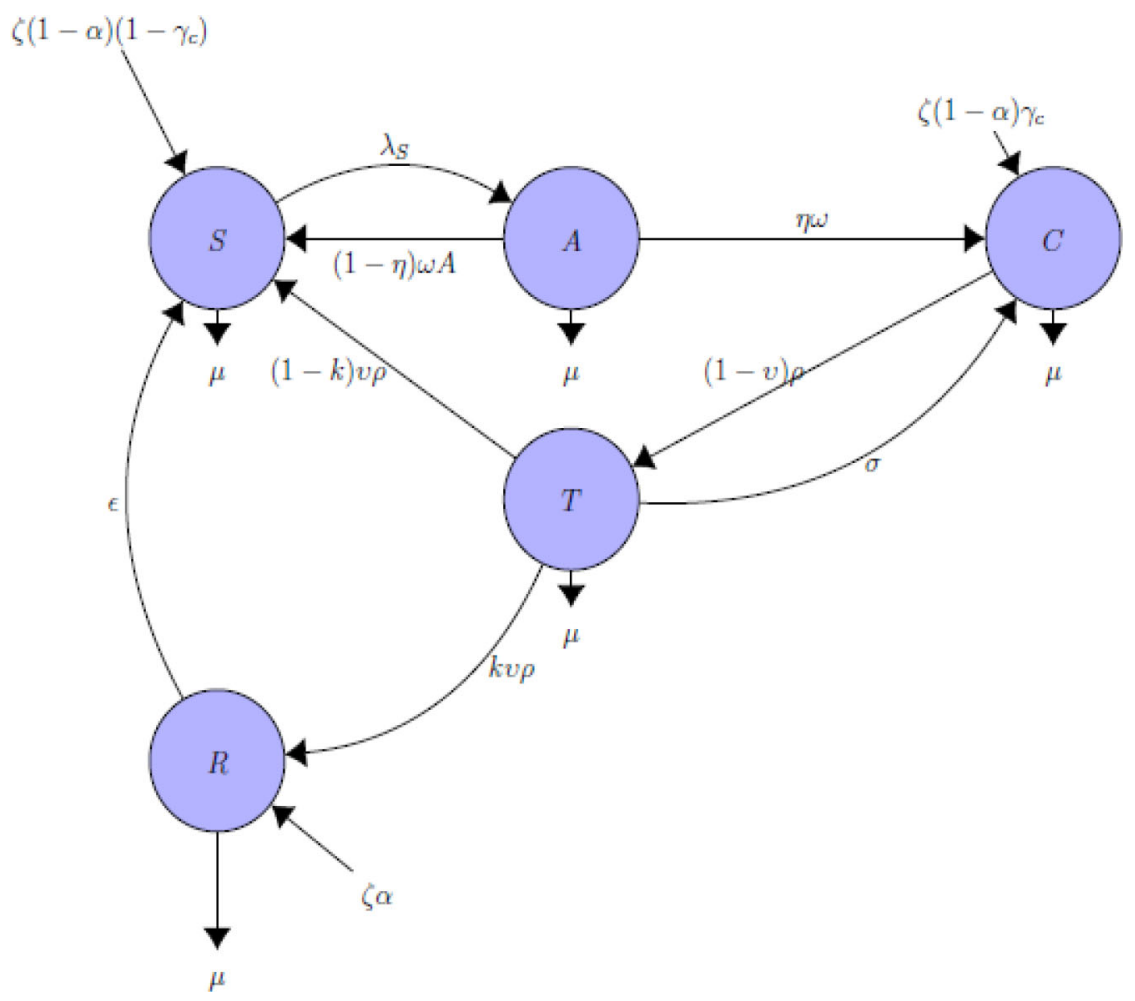

Figure 1. Compartmental flow diagram of hepatitis B virus. 
develop to chronic state. For all classes, individuals die at a constant natural mortality rate, $\mu$. We assume that HBV infected individuals on treatment are infectious. Susceptible individual $S$ may acquire HBV infection when in contact with individuals in $A, C$, and $T$ populace at a rate $\lambda_{s}$ (force of infection associated with HBV), where

$$
\lambda_{s}=\beta A+\xi_{1} \beta C+\xi_{2} \beta T
$$

where $\beta A$ and $\beta C$ are the effective contact rate for $\mathrm{HBV}$ infection to occur/probability that a contact will result in an Acute and Chronic HBV compartment, respectively. Modification $\xi>1$ accounts for a higher risk of HBV acquisition for people living with Chronic HBV.

A proportion of the acute HBV infected individuals $\eta$, becomes chronic carriers and then get treated at $\sigma$, while the remaining proportion $(1-\eta)$ spontaneously clear the virus. $\frac{1}{\omega}$ is the duration of acute phase. A proportion of the treated HBV individuals, $\kappa$, recover with full immunity, some were in the process of recovering in the treated populace at a rate, $v$ and duration for the treatment is given as $\rho$ while the remaining proportion $(1-\kappa)$ becomes susceptible. Those individuals in the process of recovering in the treated populace at a rate, $v$ if engaging/exposed to high-risk habit and those on treatment $\rho$ can be re-infected at the rate $v \rho$ if they discontinue treatment at a rate of $\varepsilon$.

$$
\begin{gathered}
\frac{d S}{d t}=\zeta(1-\alpha)(1-\gamma C)-\lambda_{s} S+(1-\eta) \omega A-\mu S+(1-k) v \rho T+\varepsilon R \\
\frac{d A}{d t}=\lambda_{s} S-\omega A-\mu A \\
\frac{d C}{d t}=\eta \omega A+\zeta(1-\alpha) \gamma C+(1-v) \rho T-\sigma C-\mu C \\
\frac{d T}{d t}=\sigma C-\rho T-\mu T \\
\frac{d R}{d t}=\zeta \alpha+k v \rho T-\varepsilon R-\mu R
\end{gathered}
$$

where $\lambda_{s}=\beta A+\xi_{1} \beta C+\xi_{2} \beta T$

\section{Model implementation}

Positivity and boundedness of solutions

From model (3), we observed that the variables are nonnegative and the solutions are non-negative for all time $t \geq 0$. The parameters used are assumed to be positive and show that the feasible solutions are bounded in the region.

Lemma 1: The initial values of the parameters are

$$
\{S(0) \geq 0, A(0) \geq 0, C(0) \geq 0, T(0) \geq 0, R(0) \geq, 0 \text { and } N(0) \geq 0\} \in \Phi
$$

Then the solution of the model $\{S(t), A(t), C(t), T(t), R(t), N(t)\}$ is positive for all $t \geq 0$.

Proof: Considering the first equation in (3),

$$
\frac{d S}{d t}=\zeta(1-\alpha)(1-\gamma C)-\lambda_{s} S+(1-\eta) \omega A-\mu S+(1-k) v \rho T+\varepsilon R
$$

we have,

$$
\begin{gathered}
\frac{d S}{d t} \geq-\left(\lambda_{s}+\mu\right) S \int \frac{1}{S} d S \geq \int-\left(\lambda_{s}+\mu\right) d t \\
S \geq S_{0} e^{-\left(\lambda_{s}+\mu\right)} \geq 0
\end{gathered}
$$

Hence, $S \geq 0$ 
With respect to the second equation in (3), we have

$$
\begin{gathered}
\frac{d A}{d t}=\lambda_{s} S-\omega A-\mu A \\
\frac{d A}{d t} \geq-(\omega+\mu) A \\
\int \frac{1}{A} d A \geq \int-(\omega+\mu) d t \\
A \geq A_{0} e^{-(\omega+\mu)} \geq 0
\end{gathered}
$$

Hence, $A \geq 0$

The same approach applies to the proof of the positivity of C, T and R.

Equilibrium points and reproduction number

The disease-free equilibrium of the model (3) exists and is given by:

$$
\left(E_{o}\right)=\left[\frac{\zeta(1-\alpha)}{\mu}, 0,0,0,0\right]
$$

The endemic steady state of the model (3) exists and is presented as follows:

$$
\begin{gathered}
S^{*}=-\left(\frac{(\mu+\omega)\left(\zeta \gamma(\mu+\rho)(\alpha-1) \rho \sigma v+\mu^{2}+\mu \rho+\mu \sigma\right) \varepsilon(\alpha \mu-\varepsilon-\mu)}{L}\right) \\
A^{*}=\left(\frac{S^{*}}{\Lambda(\mu+\omega)}\right) \\
C^{*}=-\left(\frac{(\mu+\rho) \eta \omega \Lambda \xi(\alpha \mu-\varepsilon-\mu)}{L}\right) \\
T^{*}=\left(\frac{C^{*}}{\sigma(\mu+\rho)}\right) \\
R^{*}=\left(\frac{H}{L}\right)
\end{gathered}
$$

where

$$
\begin{aligned}
L= & \mu\left(\Lambda \eta k \omega \rho \sigma v+\Lambda \zeta \alpha \varepsilon \gamma \mu+\Lambda \zeta \alpha \varepsilon \gamma \rho+\Lambda \zeta \alpha \gamma \mu^{2}+\Lambda \zeta \alpha \gamma \mu \rho+\zeta \alpha \varepsilon \gamma \mu^{2}+\zeta \alpha \varepsilon \gamma \mu \omega+\zeta \alpha \varepsilon \gamma \mu \rho+\zeta \alpha \gamma \mu^{3}+\zeta \alpha \gamma \mu^{2} \omega\right. \\
& +\zeta \alpha \gamma \mu^{2} \rho+\zeta \alpha \gamma \mu \omega \rho-\Lambda \zeta \varepsilon \gamma \mu-\Lambda \zeta \varepsilon \gamma \rho-\Lambda \zeta \gamma \mu^{2}-\Lambda \zeta \gamma \mu \rho+\Lambda \varepsilon \eta \mu \omega+\Lambda \varepsilon \eta \omega \rho+\Lambda \varepsilon \eta \omega \sigma+\Lambda \varepsilon \rho \sigma v \\
& +\Lambda \eta \mu^{2} \omega \Lambda \eta \mu \omega \rho+\Lambda \eta \mu \omega \sigma+\Lambda \mu \rho \sigma v-\zeta \varepsilon \gamma \mu^{2}-\zeta \varepsilon \gamma \mu \omega-\zeta \varepsilon \gamma \mu \rho-\zeta \gamma \mu^{3}-\zeta \gamma \mu^{2} \omega-\zeta \gamma \mu^{2} \rho-\zeta \gamma \mu \omega \rho \\
& +\varepsilon \mu \rho \sigma v+\varepsilon \omega \rho \sigma v+\mu^{2} \rho \sigma v+\mu \omega \rho \sigma v+\Lambda \varepsilon \mu^{2}+\Lambda \varepsilon \mu \rho+\Lambda \varepsilon \mu \sigma+\Lambda \mu^{3}+\Lambda \mu^{2} \rho+\Lambda \mu^{2} \sigma \\
& +\varepsilon \mu^{3}+\varepsilon \mu^{2} \rho+\varepsilon \mu^{2} \sigma+\varepsilon \mu \omega \rho+\varepsilon \mu \omega \sigma+\mu^{4}+\mu^{3} \omega+\mu^{3} \rho+\mu^{3} \sigma+\mu^{2} \omega \rho+\mu^{2} \omega \sigma \\
H= & \left.\left.\left.\left(\alpha^{2}-\alpha\right) \gamma \mu^{3}+(\Lambda+\omega+\rho) \alpha^{2}+(-\Lambda-\omega-\rho) \alpha\right) \gamma \mu^{2}+(\Lambda \rho+\omega \rho) \alpha^{2}+(-\Lambda \rho-\omega \rho) \alpha\right) \gamma \mu\right) \zeta^{2} \\
& +\left(\alpha \mu^{4}+(\rho+\omega+\sigma+\Lambda) \alpha \mu^{3}+((\sigma v+\Lambda+\omega) \rho+(\eta \omega+\sigma) \Lambda+\omega \sigma) \alpha \mu^{2}+((\eta \omega+\sigma v) \Lambda+\omega \sigma v) \rho+\Lambda \eta \omega \sigma\right) \alpha \mu \\
& +\Lambda \eta k \omega \rho \sigma v) \zeta
\end{aligned}
$$

By using the next generation matrix, the basic reproduction number is determined and given by: 


$$
\begin{gathered}
F=\left[\begin{array}{ccc}
\frac{\beta \zeta(1-\alpha)}{\mu} & \frac{\xi_{1} \beta \zeta(1-\alpha)}{\mu} & \frac{\xi_{2} \beta \zeta(1-\alpha)}{\mu} \\
0 & 0 & 0 \\
0 & 0 & 0
\end{array}\right] \\
V=\left[\begin{array}{ccc}
\omega+\mu & 0 & 0 \\
-\eta \omega & -\zeta(1-\alpha) \gamma+\sigma+\mu & 0 \\
0 & -\sigma & \rho+\mu
\end{array}\right] \\
V^{-1}=\left[\begin{array}{c}
\omega+\mu \\
\frac{\eta \omega(\rho+\mu)}{(\omega+\mu)(\zeta \alpha \gamma \mu+\zeta \alpha \gamma \rho-\zeta \gamma \mu-\zeta \gamma \rho+\rho \sigma v-\rho \sigma)} \frac{(1-v) \rho}{(\zeta \alpha \gamma \mu+\zeta \alpha \gamma \rho-\zeta \gamma \mu-\zeta \gamma \rho+\rho \sigma v-\rho \sigma)} \\
\frac{\eta \omega \sigma}{(\omega+\mu)(\zeta \alpha \gamma \mu+\zeta \alpha \gamma \rho-\zeta \gamma \mu-\zeta \gamma \rho+\rho \sigma \nu-\rho \sigma)} \frac{\sigma}{(\zeta \alpha \gamma \mu+\zeta \alpha \gamma \rho-\zeta \gamma \mu-\zeta \gamma \rho+\rho \sigma \nu-\rho \sigma)} \\
\frac{\zeta \alpha \gamma+\zeta \gamma+\mu+\rho}{(\zeta \alpha \gamma \mu+\zeta \alpha \gamma \rho-\zeta \gamma \mu-\zeta \gamma \rho+\rho \sigma v-\rho \sigma)}
\end{array}\right]
\end{gathered}
$$

The reproduction number is given by $\rho(F V-1)$, and

$$
R 0=\frac{\beta \zeta(1-\alpha)}{\mu(\omega+\mu)}+\frac{\beta \zeta(1-\alpha) \eta \omega\left(\mu \xi_{1}+\rho \xi_{1}+\sigma \xi_{2}\right)}{\mu(\omega+\mu)(\zeta \alpha \gamma \mu+\zeta \alpha \gamma \rho-\zeta \gamma \mu-\zeta \gamma \rho+\rho \sigma v-\rho \sigma)}
$$

Global stability of the equilibria

The global stability of the disease-free equilibrium was investigated using the Comparison method at the disease - free equilibrium $E_{o}$. Theorem 1 proves the global stability of disease -free equilibrium $E_{o}$

Theorem 1: The disease - free equilibrium $E_{o}$ of system (3) is globally asymptotically stable if $R_{o}<1$ and unstable if $R_{o}>1$.

Proof: The Comparison method as implemented in Lashmkantham, et al (1989) and Mushayabasa et al (2011) is used here. The rate of change of the acute and chronic components of system (3) can be written as

$$
\left(\begin{array}{l}
\frac{d A}{d t} \\
\frac{\mathrm{dC}}{d t} \\
\frac{\mathrm{dT}}{d t}
\end{array}\right)=(F-V)\left(\begin{array}{l}
A \\
C \\
T
\end{array}\right)-\left(1-\frac{S}{N}\right)
$$

where,

$$
\begin{gathered}
F=\left[\begin{array}{ccc}
\frac{\beta \zeta(1-\alpha)}{\mu} & \frac{\xi_{1} \beta \zeta(1-\alpha)}{\mu} & \frac{\xi_{2} \beta \zeta(1-\alpha)}{\mu} \\
0 & 0 & 0 \\
0 & 0 & 0
\end{array}\right] \\
V=\left[\begin{array}{ccc}
\omega+\mu & 0 & 0 \\
-\eta \omega & -\zeta(1-\alpha) \gamma+\sigma+\mu & 0 \\
0 & -\sigma & \rho+\mu
\end{array}\right]
\end{gathered}
$$

Since at the disease free $A=C=T=R=0 \rightarrow(0,0,0,0)$ and $S \leq N$ as $t \rightarrow \infty$.

Thus,

$$
\left(\begin{array}{l}
\frac{d A}{d t} \\
\frac{\mathrm{dC}}{d t} \\
\frac{\mathrm{dT}}{d t}
\end{array}\right) \leq(F-V)\left(\begin{array}{l}
A \\
C \\
T
\end{array}\right)
$$


Then all eigenvalues of the matrix $(F-V)$ have negative real parts, i.e

$$
\begin{aligned}
& {\left[\begin{array}{ccc}
\frac{\beta \zeta(1-\alpha)}{\mu}-\omega-\mu-\lambda & \frac{\xi_{1} \beta \zeta(1-\alpha)}{\mu} & \frac{\xi_{2} \beta \zeta(1-\alpha)}{\mu} \\
\eta \omega & \zeta(1-\alpha) \gamma+\sigma+\mu-\lambda & 0 \\
0 & \sigma & -(\rho+\mu)-\lambda
\end{array}\right]=0} \\
& \lambda^{3}+\left(\frac{\beta \zeta \alpha-\zeta(1-\alpha) \gamma \mu-\beta \zeta+3 \mu^{2}+\omega \mu+\rho \mu+\sigma \mu}{\mu}\right) \lambda^{2} \\
& +\frac{1}{\mu}\left(\left(\begin{array}{l}
\eta \omega \xi_{1} \beta \zeta \alpha-\zeta(1-\alpha) \gamma \zeta \alpha \beta-\eta \omega \xi_{1} \beta \zeta+\zeta(1-\alpha) \gamma \beta \zeta-2 \zeta(1-\alpha) \gamma \mu^{2}-\zeta(1-\alpha) \gamma \mu \omega-\zeta(1-\alpha) \gamma \mu \rho+2 \zeta \alpha \beta \mu \\
+\zeta \alpha \beta \rho+\zeta \alpha \beta \sigma-2 \zeta \beta \mu-\zeta \beta \rho-\zeta \beta \sigma+3 \mu^{3}+2 \mu^{2} \omega+2 \mu^{2} \rho+2 \mu^{2} \sigma+\mu \omega \rho+\mu \omega \sigma+\mu \rho \sigma
\end{array}\right)\right) \lambda \\
& +\frac{1}{\mu}\left(\begin{array}{l}
\zeta \alpha \beta \rho \mu+\zeta \alpha \beta \mu \sigma+\zeta \alpha \beta \rho \sigma+\zeta(1-\alpha) \gamma \beta \zeta \mu+\zeta(1-\alpha) \gamma \beta \zeta \rho-\zeta(1-\alpha) \gamma \mu \omega \rho-\eta \mu \omega \xi_{1} \beta \zeta-\eta \omega \xi_{1} \beta \zeta \rho \\
-\zeta(1-\alpha) \gamma \zeta \alpha \beta \mu-\zeta(1-\alpha) \gamma \zeta \alpha \beta \rho+\mu^{4}-\beta \zeta \mu^{2}+\mu^{2} \omega \rho+\mu^{2} \omega \sigma+\mu^{2} \rho \sigma-\zeta(1-\alpha) \gamma \mu^{3}+\mu^{3} \omega+\mu^{3} \rho+\mu^{3} \sigma \\
+\zeta \alpha \beta \mu^{2}-\zeta \beta \rho \mu-\zeta \beta \mu \sigma-\zeta \beta \rho \sigma+\mu \omega \rho \sigma-\zeta(1-\alpha) \gamma \mu^{2} \omega-\zeta(1-\alpha) \gamma \mu^{2} \rho-\eta \omega \xi_{2} \beta \zeta \sigma+\alpha \eta \omega \xi_{2} \beta \zeta \sigma \\
+\eta \omega \xi_{1} \beta \zeta \alpha \mu+\eta \omega \mu \rho \xi_{1} \beta \zeta
\end{array}\right)
\end{aligned}
$$

Equation (11) has three negative roots by Descartes rule of signs if

$$
\begin{aligned}
& \frac{1}{\mu}\left(\begin{array}{l}
\zeta \alpha \beta \rho \mu+\zeta \alpha \beta \mu \sigma+\zeta \alpha \beta \rho \sigma+\zeta(1-\alpha) \gamma \beta \zeta \mu+\zeta(1-\alpha) \gamma \beta \zeta \rho-\zeta(1-\alpha) \gamma \mu \omega \rho-\eta \mu \omega \xi_{1} \beta \zeta-\eta \omega \xi_{1} \beta \zeta \rho \\
-\zeta(1-\alpha) \gamma \zeta \alpha \beta \mu-\zeta(1-\alpha) \gamma \zeta \alpha \beta \rho+\mu^{4}-\beta \zeta \mu^{2}+\mu^{2} \omega \rho+\mu^{2} \omega \sigma+\mu^{2} \rho \sigma-\zeta(1-\alpha) \gamma \mu^{3}+\mu^{3} \omega+\mu^{3} \rho+\mu^{3} \sigma \\
+\zeta \alpha \beta \mu^{2}-\zeta \beta \rho \mu-\zeta \beta \mu \sigma-\zeta \beta \rho \sigma+\mu \omega \rho \sigma-\zeta(1-\alpha) \gamma \mu^{2} \omega-\zeta(1-\alpha) \gamma \mu^{2} \rho-\eta \omega \xi_{2} \beta \zeta \sigma+\alpha \eta \omega \xi_{2} \beta \zeta \sigma \\
+\eta \omega \xi_{1} \beta \zeta \alpha \mu+\eta \omega \mu \rho \xi_{1} \beta \zeta
\end{array}\right)
\end{aligned}
$$

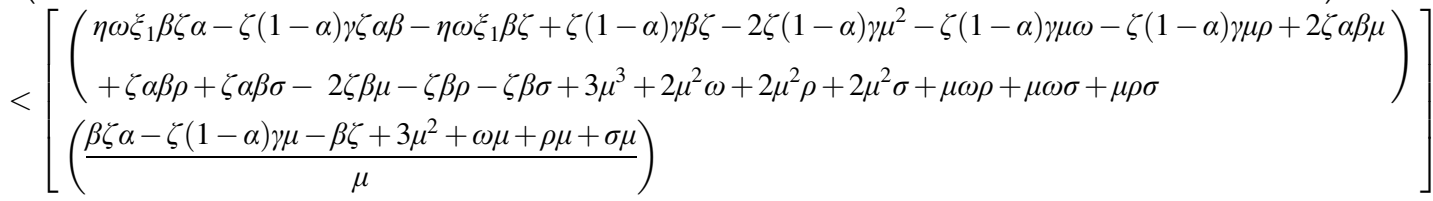

$$
\begin{aligned}
& \zeta \alpha \beta \rho \mu+\zeta \alpha \beta \mu \sigma+\zeta \alpha \beta \rho \sigma+\zeta(1-\alpha) \gamma \beta \zeta \mu+\zeta(1-\alpha) \gamma \beta \zeta \rho-\zeta(1-\alpha) \gamma \mu \omega \rho-\eta \mu \omega \xi_{1} \beta \zeta-\eta \omega \xi_{1} \beta \zeta \rho \\
& -\zeta(1-\alpha) \gamma \zeta \alpha \beta \mu-\zeta(1-\alpha) \gamma \zeta \alpha \beta \rho+\mu^{4}-\beta \zeta \mu^{2}+\mu^{2} \omega \rho+\mu^{2} \omega \sigma+\mu^{2} \rho \sigma-\zeta(1-\alpha) \gamma \mu^{3}+\mu^{3} \omega+\mu^{3} \rho+\mu^{3} \sigma \\
& +\zeta \alpha \beta \mu^{2}-\zeta \beta \rho \mu-\zeta \beta \mu \sigma-\zeta \beta \rho \sigma+\mu \omega \rho \sigma-\zeta(1-\alpha) \gamma \mu^{2} \omega-\zeta(1-\alpha) \gamma \mu^{2} \rho-\eta \omega \xi_{2} \beta \zeta \sigma+\alpha \eta \omega \xi_{2} \beta \zeta \sigma \\
& \frac{+\eta \omega \xi_{1} \beta \zeta \alpha \mu+\eta \omega \mu \rho \xi_{1} \beta \zeta}{\left(\begin{array}{l}
\eta \omega \xi_{1} \beta \zeta \alpha-\zeta(1-\alpha) \gamma \zeta \alpha \beta-\eta \omega \xi_{1} \beta \zeta+\zeta(1-\alpha) \gamma \beta \zeta-2 \zeta(1-\alpha) \gamma \mu^{2}-\zeta(1-\alpha) \gamma \mu \omega-\zeta(1-\alpha) \gamma \mu \rho+2 \zeta \alpha \beta \mu \\
+\zeta \alpha \beta \rho+\zeta \alpha \beta \sigma-2 \zeta \beta \mu-\zeta \beta \rho-\zeta \beta \sigma+3 \mu^{3}+2 \mu^{2} \omega+2 \mu^{2} \rho+2 \mu^{2} \sigma+\mu \omega \rho+\mu \omega \sigma+\mu \rho \sigma
\end{array}\right)}<1 \\
& \left(\beta \zeta \alpha-\zeta(1-\alpha) \gamma \mu-\beta \zeta+3 \mu^{2}+\omega \mu+\rho \mu+\sigma \mu\right)
\end{aligned}
$$

$$
\therefore R_{0}<1 \text {. }
$$

It follows that the linearized differential inequality is stable whenever $R_{0}<1$. Consequently, $(A, C, T) \rightarrow(0,0,0)$ as $t \rightarrow \infty$. Evaluating system (3) at $A=C=T=0$ gives $S \rightarrow 1$ for $R_{0}<1$. Hence, the disease-free equilibrium $E_{0}$ of system (3) is globally asymptotically stable if $R_{0}<1$. The result also follows immediately that the disease-free equilibrium $E_{0}$ of system (3) is unstable if $R_{0}>1$.

Theorem 2: The equations of the model has a positive distinctive endemic equilibrium whenever R0 $>1$, which is said to be globally asymptotically stable.

Proof: Considering the Lyapunov function defined as:

$$
\begin{aligned}
L\left(S^{*}, A^{*}, C^{*}, T^{*}, R^{*}\right)= & \left(S-S^{*} \ln \left(\frac{S}{S^{*}}\right)\right)+\left(A-A^{*} \ln \left(\frac{A}{A^{*}}\right)\right)+\left(C-C^{*} \ln \left(\frac{C}{C^{*}}\right)\right)+\left(T-T^{*} \ln \left(\frac{T}{T^{*}}\right)\right) \\
& +\left(R-R^{*} \ln \left(\frac{R}{R^{*}}\right)\right)
\end{aligned}
$$


where $\mathrm{L}$ takes it derivative along the system directly as:

$$
\begin{aligned}
\frac{d L}{d t}=\left(1-\frac{S^{*}}{S}\right) \frac{d S}{d t}+\left(1-\frac{A^{*}}{A}\right) \frac{d A}{d t}+\left(1-\frac{C^{*}}{C}\right) \frac{d C}{d t}+\left(1-\frac{T^{*}}{T}\right) \frac{d T}{d t}+\left(1-\frac{R^{*}}{R}\right) \frac{d R}{d t} \\
\frac{d L}{d t}=\left(1-\frac{S^{*}}{S}\right)\left[\zeta(1-\alpha)(1-\gamma C)-\left(\beta A+\xi_{1} \beta C+\xi_{2} \beta T\right) S+(1-\eta) \omega A-\mu S+(1-k) v \rho T+\varepsilon R\right] \\
+\left(1-\frac{A^{*}}{A}\right)\left[\left(\beta A+\xi_{1} \beta C+\xi_{2} \beta T\right) S-(\omega+\mu) A\right]+\left(1-\frac{C^{*}}{C}\right)[\eta \omega A+(1-v) \rho T-(\sigma+\mu-\zeta(1-\alpha) \gamma) C] \\
+\left(1-\frac{T^{*}}{T}\right)[\sigma C-(\rho+\mu) T]+\left(1-\frac{R^{*}}{R}\right)[\zeta \alpha+k v \rho T-(\varepsilon+\mu) R]
\end{aligned}
$$

At equilibrium,

$$
\begin{aligned}
& \left.\zeta(1-\alpha)(1-\gamma C)=\left(\beta A^{*}+\xi_{1} \beta C^{*}+\xi_{2} \beta T^{*}\right) S^{*}-(1-\eta) \omega A^{*}+\mu S^{*}-(1-k) v \rho T^{*}-\varepsilon R^{*}\right) \\
& (\omega+\mu)=\frac{\left(\beta A^{*}+\xi_{1} \beta C^{*}+\xi_{2} \beta T^{*}\right) S^{*}}{A^{*}} \\
& (\sigma+\mu-\zeta(1-\alpha) \gamma)=\frac{\eta \omega A^{*}+(1-v) \rho T^{*}}{C^{*}} \\
& (\rho+\mu)=\frac{\sigma C^{*}}{T^{*}} \\
& (\varepsilon+\mu) R=\frac{\zeta \alpha+k v \rho T^{*}}{R^{*}} \\
& \frac{d L}{d t}=\left(1-\frac{S^{*}}{S}\right)\left[\begin{array}{l}
\left(\beta A^{*}+\xi_{1} \beta C^{*}+\xi_{2} \beta T^{*}\right) S^{*}-(1-\eta) \omega A^{*}+\mu S^{*}-(1-k) v \rho T^{*}-\varepsilon R^{*} \\
-\left(\beta A+\xi_{1} \beta C+\xi_{2} \beta T\right) S+(1-\eta) \omega A-\mu S+(1-k) v \rho T+\varepsilon R
\end{array}\right] \\
& +\left(1-\frac{A^{*}}{A}\right)\left[\left(\beta A+\xi_{1} \beta C+\xi_{2} \beta T\right) S-\frac{\left(\beta A^{*}+\xi_{1} \beta C^{*}+\xi_{2} \beta T^{*}\right) S^{*} A}{A^{*}}\right] \\
& +\left(1-\frac{C^{*}}{C}\right)\left[\eta \omega A+(1-v) \rho T-\frac{\eta \omega A^{*}+(1-v) \rho T^{*} C}{C^{*}}\right]+\left(1-\frac{T^{*}}{T}\right)\left[\sigma C-\frac{\sigma C^{*} T}{T^{*}}\right] \\
& +\left(1-\frac{R^{*}}{R}\right)\left[\zeta \alpha+k v \rho T-\frac{\zeta \alpha+k v \rho T^{*} R}{R^{*}}\right] \\
& =\left(1-\frac{S^{*}}{S}\right)\left[\begin{array}{l}
\beta A^{*} S^{*}+\xi_{1} \beta C^{*} S^{*}+\xi_{2} \beta T^{*} S^{*}-(1-\eta) \omega A^{*}+\mu S^{*}-(1-k) v \rho T^{*} \\
-\varepsilon R^{*}-\beta A S-\xi_{1} \beta C S-\xi_{2} \beta T S+(1-\eta) \omega A-\mu S+(1-k) v \rho T+\varepsilon R
\end{array}\right] \\
& +\left(1-\frac{A^{*}}{A}\right)^{[}\left[\beta A S+\xi_{1} \beta C S+\xi_{2} \beta T S-\frac{\beta A^{*} S^{*} A}{A^{*}}-\frac{\xi_{1} \beta C^{*} S^{*} A}{A^{*}}-\frac{\xi_{2} \beta T^{*} S^{*} A}{A^{*}}\right]+\left(1-\frac{C^{*}}{C}\right) \eta \omega A+(1-v) \rho T\left[1-\frac{A^{*} T^{*} C}{\mathrm{ATC}^{*}}\right] \\
& +\left(1-\frac{T^{*}}{T}\right) \sigma C\left[1-\frac{C^{*} T}{C T^{*}}\right]+\left(1-\frac{R^{*}}{R}\right) \zeta \alpha+k v \rho T\left[1-\frac{T^{*} R}{T R^{*}}\right] \\
& =\left(1-\frac{S^{*}}{S}\right)\left[\begin{array}{l}
-\beta A S\left(1-\frac{A^{*} S^{*}}{A S}\right)-\xi_{1} \beta C S\left(1-\frac{C^{*} S^{*}}{C S}\right)-\xi_{2} \beta T S\left(1-\frac{T^{*} S^{*}}{T S}\right)+(1-\eta) \omega A\left(1-\frac{A^{*}}{A}\right) \\
-\mu S\left(1-\frac{S^{*}}{S}\right)+(1-k) v \rho T\left(1-\frac{T^{*}}{T}\right)+\varepsilon R\left(1-\frac{R^{*}}{R}\right)
\end{array}\right] \\
& +\left(1-\frac{A^{*}}{A}\right)\left[\beta A S\left(1-\frac{A^{*} S^{*}}{S A^{*}}\right)+\xi_{1} \beta C S\left(1-\frac{C^{*} S^{*} A}{S C A^{*}}\right)+\xi_{2} \beta T S\left(1-\frac{T^{*} S^{*} A}{T S A^{*}}\right)\right]+\eta \omega A \\
& +(1-v) \rho T\left(1-\frac{C^{*}}{C}\right)\left[1-\frac{A^{*} T^{*} C}{\mathrm{AT} C^{*}}\right]+\sigma C\left(1-\frac{T^{*}}{T}\right)\left[1-\frac{C^{*} T}{C T^{*}}\right]+\zeta \alpha+k v \rho T\left(1-\frac{R^{*}}{R}\right)\left[1-\frac{T^{*} R}{T R^{*}}\right]
\end{aligned}
$$




$$
\begin{gathered}
=-\mu S\left(1-\frac{S^{*}}{S}\right)^{2}-\beta A S\left(1-\frac{S^{*}}{S}\right)\left(1-\frac{A^{*} S^{*}}{A S}\right)-\xi_{1} \beta C S\left(1-\frac{S^{*}}{S}\right)\left(1-\frac{C^{*} S^{*}}{C S}\right)-\xi_{2} \beta T S\left(1-\frac{S^{*}}{S}\right)\left(1-\frac{T^{*} S^{*}}{T S}\right) \\
+(1-\eta) \omega A\left(1-\frac{S^{*}}{S}\right)\left(1-\frac{A^{*}}{A}\right)+(1-k) v \rho T\left(1-\frac{S^{*}}{S}\right)\left(1-\frac{T^{*}}{T}\right)+\varepsilon R\left(1-\frac{S^{*}}{S}\right)\left(1-\frac{R^{*}}{R}\right) \\
+\beta A S\left(1-\frac{A^{*}}{A}\right)\left(1-\frac{A^{*} S^{*}}{S A^{*}}\right)+\xi_{1} \beta C S\left(1-\frac{A^{*}}{A}\right)\left(1-\frac{C^{*} S^{*} A}{S C A^{*}}\right)+\xi_{2} \beta T S\left(1-\frac{A^{*}}{A}\right)\left(1-\frac{T^{*} S^{*} A}{T S A^{*}}\right)+\eta \omega A \\
+(1-v) \rho T\left(1-\frac{C^{*}}{C}\right)\left(1-\frac{A^{*} T^{*} C}{A T C^{*}}\right)+\sigma C\left(1-\frac{T^{*}}{T}\right)\left(1-\frac{C^{*} T}{C T^{*}}\right)+\zeta \alpha+k v \rho T\left(1-\frac{R^{*}}{R}\right)\left(1-\frac{T^{*} R}{T R^{*}}\right) \\
=-\mu S\left(1-\frac{S^{*}}{S}\right)^{2}+P_{1}(S, A, C, T, R)+P_{2}(S, A, C, T, R)
\end{gathered}
$$

where,

$$
\begin{aligned}
P_{1}(S, A, C, T, R)= & -\beta A S\left(1-\frac{S^{*}}{S}\right)\left(1-\frac{A^{*} S^{*}}{A S}\right)-\xi_{1} \beta C S\left(1-\frac{S^{*}}{S}\right)\left(1-\frac{C^{*} S^{*}}{C S}\right)-\xi_{2} \beta T S\left(1-\frac{S^{*}}{S}\right)\left(1-\frac{T^{*} S^{*}}{T S}\right) \\
P_{2}(S, A, C, T, R)= & (1-\eta) \omega A\left(1-\frac{S^{*}}{S}\right)\left(1-\frac{A^{*}}{A}\right)+(1-k) v \rho T\left(1-\frac{S^{*}}{S}\right)\left(1-\frac{T^{*}}{T}\right)+\varepsilon R\left(1-\frac{S^{*}}{S}\right)\left(1-\frac{R^{*}}{R}\right) \\
& +\beta A S\left(1-\frac{A^{*}}{A}\right)\left(1-\frac{A^{*} S^{*}}{S A^{*}}\right)+\xi_{1} \beta C S\left(1-\frac{A^{*}}{A}\right)\left(1-\frac{C^{*} S^{*} A}{S C A^{*}}\right)+\xi_{2} \beta T S\left(1-\frac{A^{*}}{A}\right)\left(1-\frac{T^{*} S^{*} A}{T S A^{*}}\right) \\
& +\eta \omega A+(1-v) \rho T\left(1-\frac{C^{*}}{C}\right)\left(1-\frac{A^{*} T^{*} C}{\mathrm{ATC}}\right)+\sigma C\left(1-\frac{T^{*}}{T}\right)\left(1-\frac{C^{*} T}{C T^{*}}\right)+\zeta \alpha \\
& +k v \rho T\left(1-\frac{R^{*}}{R}\right)\left(1-\frac{T^{*} R}{T R^{*}}\right)
\end{aligned}
$$

$P_{1} \leq 0$ whenever

$$
A S \geq A^{*} S^{*}, C S \geq C^{*} S^{*}, T S \geq T^{*} S^{*}
$$

and

$P_{2} \leq 0$ whenever

$$
A^{*} S \geq A^{*} S^{*}, A^{*} C S \geq A C^{*} S^{*}, T S A^{*} \geq T^{*} S^{*} A, A T C^{*} \geq A^{*} T^{*} C, C T^{*} \geq C^{*} T, T R^{*} \geq T^{*} R
$$

Thus,

$\frac{d L}{d t} \leq 0$ if the condition in (19) and (20) holds.

Therefore, by LaSalle asymptotic stability theorem (LaSalle, 1976), and Adeniyi et al. (2020), the positive equilibrium state $\frac{d L}{d t}$ is globally asymptotically stable in the positive region $R_{+}^{5}$.

\section{Sensitivity indices}

To test the strength of the model and the parameter values, a sensitivity study was carried out. This is done in order know the parameters that have a huge influence on the basic reproduction number (R0) which is done using Maple 19 software. A variable $\mathrm{k}$; a normalized forward sensitivity index which depends on a parameter: $\ell$ differentially, is defined as:

$$
\hbar_{\ell}^{k}=\frac{\partial k \ell}{\partial \ell} \frac{\ell}{k}
$$

The R0 sensitivity is therefore derived from each of the different parameters listed in Table 1. All expressions are complex for sensitivity indices, so sensitivity indices are evaluated in Table 2 at the baseline parameter values.

\section{Model validation}

To validate our analytical results, we perform numerical simulations of the proposed model (2). These simulations are based on qualitative analysis. Some of the parameters were obtained from published research, while others were estimated 
Table 2. Sensitivity indices on R0.

\begin{tabular}{|l|l|}
\hline Parameter & Sensitivity index \\
\hline$\zeta$ & 1.0000041 \\
\hline$\beta$ & 0.9999999 \\
\hline$\xi$ & 0.0027767 \\
\hline$\epsilon$ & 0.3456467 \\
\hline$\eta$ & 0.0027766 \\
\hline$\gamma$ & 0.0000004 \\
\hline$\sigma$ & 0.0026593 \\
\hline$\alpha$ & -0.054285 \\
\hline$\mu$ & -1.387192 \\
\hline$\omega$ & -0.955709 \\
\hline
\end{tabular}

Table 3. Parameter values used for the numerical simulation.

\begin{tabular}{|l|l|l|}
\hline Parameter & Values & Source \\
\hline$\zeta$ & 0.012100 & Khan et al (2019) \\
\hline$\beta$ & 0.009500 & Khan et al (2019) \\
\hline$\xi$ & 0.160000 & Khan et al (2019) \\
\hline$\epsilon$ & 0.050000 & Estimated \\
\hline$\eta$ & 0.067000 & Estimated \\
\hline$\gamma$ & 0.110000 & Khan et al (2019) \\
\hline$\sigma$ & 0.590000 & Khan et al (2019) \\
\hline$\alpha$ & 0.320000 & Khan et al (2019) \\
\hline$\mu$ & 0.006930 & Khan et al (2019) \\
\hline$\omega$ & 0.160000 & Estimated \\
\hline$k$ & 0.300000 & Estimated \\
\hline$\rho$ & 0.005000 & Estimated \\
\hline$v$ & 0.05000 & Khan et al (2019) \\
\hline
\end{tabular}

by the authors as they were thought to be biologically feasible. We employ a strictly numerical RK (Runge-Kutta) technique of order four embedded in the Maple 19 software. Table 3 contains the parameter's comprehensive values.

Considering the first sizes of compartmental population, taking the parameter values and the interval (0-60) using the linear stability analysis, we perform the simulations and obtain the outcomes shown in Figure 2. The dynamic behavior of susceptible individuals is represented in Figure 2A, showing the existence of the susceptible individuals. It was discovered from Figure 2B that the acute populace decreases with the passage of time. Ditto the behaviors of chronic, treated and recovered populace, respectively, are dynamically represented in Figure 2C, D and E. The trajectories $\mathrm{S}(\mathrm{t})$, $\mathrm{A}(\mathrm{t}), \mathrm{C}(\mathrm{t}), \mathrm{T}(\mathrm{t})$, and $\mathrm{R}(\mathrm{t})$ distinctly converge to the disease-free equilibrium of $\mathrm{E} 0=\left(\mathrm{S} \_0,0,0,0,0, \mathrm{R} \_0\right)=(1.678018396$, $0,0,0,0,0,0,0.06801334973$ ) as indicated in Eq. (10), when $\mathrm{R} 0=0.07150316516<1$. The dynamics of the susceptible populace with respect to the treatment rate $\sigma$, recovering rate with full immunity $\mathrm{k}$ and the rate at which recovered individual fallout from risk reduction $\epsilon$ is shown in Figure 3A. It is evident from Figure 3A that with the increase in the parametric values, the susceptible population increases, even as increase is not evident due to those who recover with complete immunity. However, there is still an increase due to some people who fall out of risk reduction, checking the cumulative impact of the parameters causing the increment of the susceptible populations. The inverse relationship of the compartmental population (acute and chronic) with the variance of the above parameters is shown in Figure 3B and C. This means that it is possible to minimize acute and chronic individuals by increasing the parametric values. The variation 

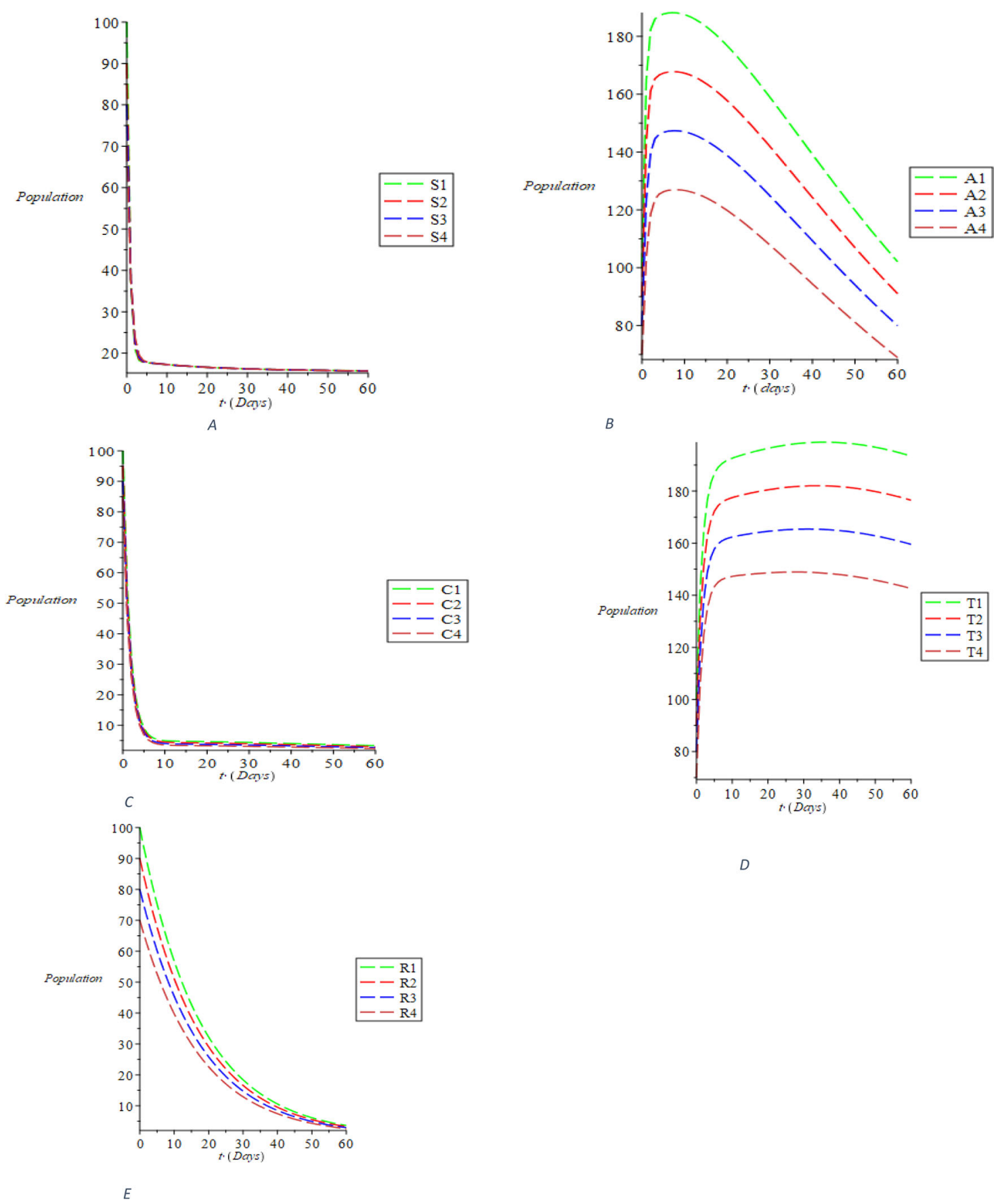

Figure 2. The dynamical behavior of the varying population of the classes: (A) susceptible (B) acute (C) chronic (D) treated (E) recovered using the Maple 19 software.

of the treated and recovered populace is shown in Figure 3D and E. An increase in the treated and the recovered population is caused by increasing the values of the parameters. It can be clearly inferred from our computational simulations that treatment, spontaneous clearance and reduction of the risk factor are highly successful in transmitting and regulating HBV transmission. The effective measure of these parameters as substantiated by the simulations is an excellent control method of the transmissible infection of HBV. 


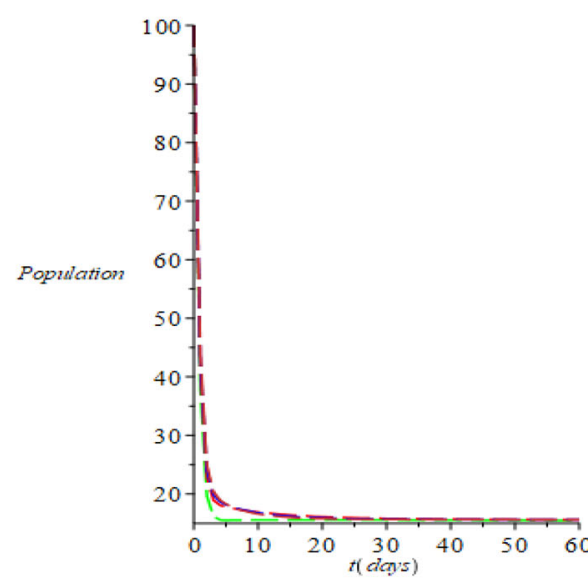

(A)

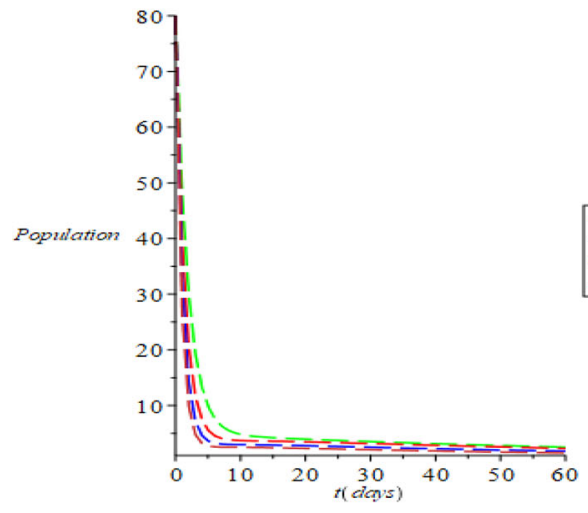

(C)

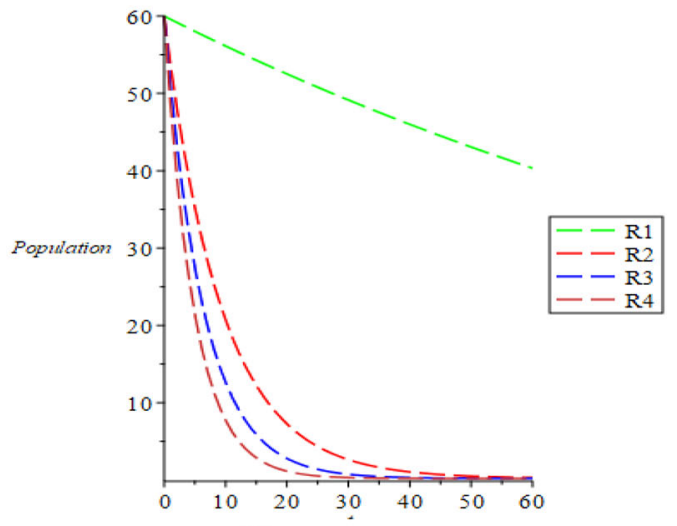

(E)

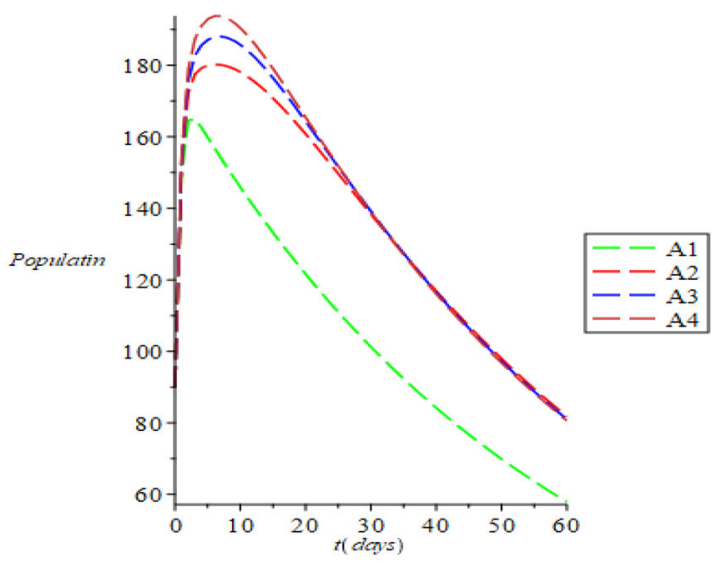

(B)

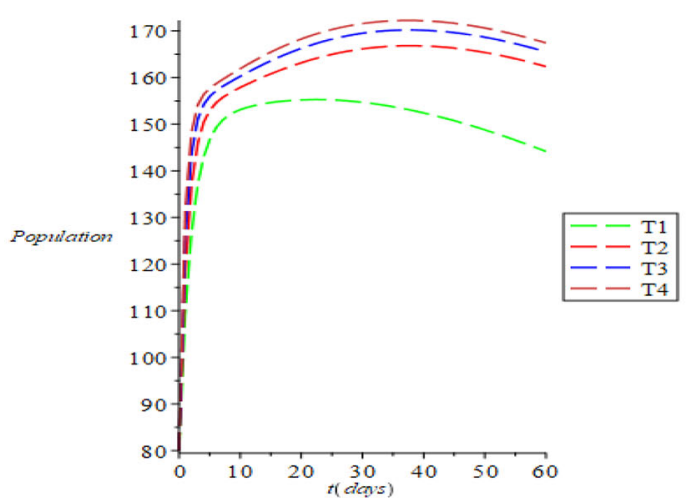

(D)

Figure 3. The dynamical behavior of the various classes varying various treatment parameter (A) susceptible (B) acute (C) chronic (D) treated (E) recovered using the Maple 19 software.

\section{Conclusion}

A deterministic model of hepatitis B that involves the spontaneous clearance of an acute individual and also recovery of chronic individual with full immunity and risk factor reduction was developed and investigated. Disease-free and endemic equilibria of the model exist. The basic reproduction number was constructed by the method of next generation matrix. The global stability of the disease-free and endemic equilibria was discussed and shown to be asymptotically stable. The effects of the treatment rate, the recovery rate with complete immunity, and the risk mitigation factor were thoroughly discussed. Future work may include using the optimum control theory to mitigate hepatitis B infection. 
Data availability

All data underlying the results are available as part of the article and no additional source data are required.

References

Castillo-Chavez C, Song B: Dynamical models of tuberculosis and their applications. Math. Biosci. Eng. 2004; 1(2): 361-404.

PubMed Abstract | Publisher Full Text

CDC: see forHepatitis B Fact sheet No. 2, 2019.

Reference Source

Ciupe SM, Ribeiro RM, Nelson PW, et al.: Modeling the mechanisms of acute hepatitis B virus infection. J Theor Biol. 2007; 247(1): 23-35.

PubMed Abstract | Publisher Full Text | Free Full Text

Fatehi Chenar F, Kyrychko YN, Blyuss KB: Mathematical model of immune response to hepatitis B. J Theor Biol. 2018; 447(March):

98-110.

PubMed Abstract | Publisher Full Text

Khan T, Ahmad S, Zaman G: Modeling and qualitative analysis of a

hepatitis B epidemic model. Chaos. 2019; 29(10).

PubMed Abstract | Publisher Full Text

Lau GKK, Cooksley H, Ribeiro RM, et al.: Impact of early viral kinetics on $\mathrm{T}$-cell reactivity during antiviral therapy in chronic hepatitis $\mathrm{B}$.

Antivir Ther. 2007; 12(5): 705-718.

Reference Source |PubMed Abstract

LaSalle JP: The Stability of Dynamical Systems, Regional Conference Series in Applied Mathematics, SIAM, Philadelphia, Pa,USA. 1976.

Long C, Qi H, Huang SH: Mathematical modeling of cytotoxic lymphocyte-mediated immune response to hepatitis $B$ virus infection. J Biomed Biotechnol. 2008; 2008: 1.

PubMed Abstract | Publisher Full Text | Free Full Text

Mpeshe SC, Nyerere N: Modeling approach to assess the transmission dynamics of Hepatitis B infection in Africa Modeling approach to assess the transmission dynamics of Hepatitis B infection in Africa. March 2019.

Pang J, Cui JA, Zhou X: Dynamical behavior of a hepatitis B virus transmission model with vaccination. J Theor Biol. 2010; 265(4): 572-578.

PubMed Abstract | Publisher Full Text

Pan CQ, Zhang JX: Natural History and Clinical

Consequences of Hepatitis B Virus Infection. Int J Med Sci. 2005; 2(1):

36-40.

Reference Source | PubMed Abstract | Publisher Full Text | Free Full

Text

van den Driessche $P$, Watmough J: Reproduction number and subthreshold endemic equilibria for compartmental models of disease transmission. Math. Biosci. 2002; 180, 29-48.

PubMed Abstract | Publisher Full Text

Qesmi R, Wu J, Wu J, et al.: Influence of backward bifurcation

in a model of hepatitis B and C viruses. Math Biosci. 2010; 224(2):

118-125.

PubMed Abstract | Publisher Full Text

Wang K, Fan A, Torres A: Global properties of an improved hepatitis B virus model. Nonlinear Analysis: Real World Applications. 2010; 11(4): 3131-3138.

Publisher Full Text

WHO: see for Hepatitis B Fact sheet No. 204. 2019

Reference Source

WHO: see for Hepatitis B Fact sheet No. 133. 2020

Reference Source 


\section{Open Peer Review}

\section{Current Peer Review Status:}

\section{Version 1}

Reviewer Report 16 August 2021

\section{https://doi.org/10.5256/f1000research.56106.r90565}

(C) 2021 Abolarinwa A. This is an open access peer review report distributed under the terms of the Creative Commons Attribution License, which permits unrestricted use, distribution, and reproduction in any medium, provided the original work is properly cited.

\section{Abimbola Abolarinwa \\ Department of Mathematics, University of Lagos, Lagos, Nigeria}

The authors develop and investigate the dynamic of HBV-model incorporating spontaneous clearance of acute individuals and recovery pf chronic individuals with full immunity. The model is subjected to sensitivity and stability analysis.

The results are well presented and discussed. I can recommend this paper for indexing if the authors can consider these suggestions:

1. Under Abstract - change positivity solution to positivity of solutions

2. Under Introduction - line 3 - change reporting to reported. Paragraph 4 - should be put in proper perspective to show the gap which this paper is trying to bridge. In my understanding, testing and treatment have always been part of epidemic (including HBV) control. You can advertise the novelty introduced in the paper. The statement "The aim of this study is to contribute ....." can be recast to make the aim measurable and specific to indicate what and what is being contributed and the its importance.

3. Under model implementation.

Lemma 1 -

Start the statement with "Suppose the initial values are ..." No need for the word parameters

No need for \$lin \Phi\$ or \$IPhi\$ should be defined

The solution of the model (3) $\{\ldots\}$ is posively invariant for all $t>0$. (not greater than or equal to).

Under the proof

The last three line on page 4(so also the first 4 lines on page 5) should be corrected

These two lines can help in each case 
"we have

\$\$frac $\{\mathrm{dS}\}\{\mathrm{dt}\} \backslash \mathrm{ge}-(\mathrm{Vlamda} s+\backslash \mathrm{mu}) \mathrm{S} \$ \$$

and

$\$ \$ S(t) \backslash g e$ s_0 $e^{\wedge}\{-\backslash l a m b d a+\backslash m u) t . \$(t$ should be included in power of e)

where $\$ s_{-} 0=S(0)$ Ige 0 \$.

Hence $S(t)>0$ for all $t>0$.

Is the work clearly and accurately presented and does it cite the current literature?

Yes

Is the study design appropriate and is the work technically sound?

Yes

Are sufficient details of methods and analysis provided to allow replication by others? Yes

If applicable, is the statistical analysis and its interpretation appropriate?

Not applicable

Are all the source data underlying the results available to ensure full reproducibility? Partly

Are the conclusions drawn adequately supported by the results?

Yes

Competing Interests: No competing interests were disclosed.

Reviewer Expertise: Differential equations, Differential Geometry

I confirm that I have read this submission and believe that I have an appropriate level of expertise to confirm that it is of an acceptable scientific standard.

Author Response 15 Oct 2021

\section{Olajumoke Oludoun}

All the comments have been attended to in the new version submitted

Competing Interests: No competing interests were disclosed. 
The benefits of publishing with F1000Research:

- Your article is published within days, with no editorial bias

- You can publish traditional articles, null/negative results, case reports, data notes and more

- The peer review process is transparent and collaborative

- Your article is indexed in PubMed after passing peer review

- Dedicated customer support at every stage

For pre-submission enquiries, contact research@f1000.com 